\title{
Bone changes in polycythaemia vera and myelosclerosis
}

\author{
B. E. ROBERTS, C. G. WOODS, D. W. MILES, AND C. R. PATERSON \\ From the University Departments of Haematology, Bone Pathology, Medicine and Clinical Investigation \\ The General Infirmary, Leeds
}

SYNOPSIS To study the problem of new bone formation in myelosclerosis bone biopsies have beeno performed in cases of polycythaemia vera, which may be regarded as a pre-myelosclerotic condition, 6 and in primary myelosclerosis; morphological studies of bone and reticulin were made and osteoido and bone matrix was quantitatively assessed. Relevant metabolic studies were also carried out, but were predominantly normal. No new bone formation was detected in polycythaemia vera and somez bone was osteoporotic; no significant increase in osteoid was found. With the use of polarized light? it was found that new bone formation in myelosclerosis resulted from the ossification of wavy argyrophilic fibres in marrow: the presence of a dense network of these wavy fibres was a pre-D requisite of new bone formation and ossification of the non-refractile branched reticulin fibres was $\overrightarrow{\mathscr{C}}$ not observed.

'Myelosclerosis' is the term commonly used to describe a haematological syndrome the main features of which are a leucoerythroblastic anaemia and hepatosplenomegaly due to extramedullary haemopoiesis. It refers in particular to the histological features which comprise fibrosis of the bone marrow (myelofibrosis) with specifically, according to Vaughan (1936) who introduced the term, excess bone in the spongiosa and medullary cavity.

Myelosclerosis may be primary in origin or it may follow polycythaemia vera. According to Szur and Lewis(1966), myelosclerosis may be the most frequent terminal syndrome in a large series of cases of polycythaemia vera followed up for a long time.

In an attempt to observe the histological changes during the transformation of polycythaemia vera to myelosclerosis trephine biopsies have been performed in a series of patients with polycythaemia vera during the various stages of the disease. The histological criteria of myelosclerosis, which were established from five myelosclerotic trephine specimens, were then sought in the sections from these patients and measurements of osteoid made. Appropriate biochemical investigations were also performed.

PATIENTS, METHODS, AND MATERIALS

The patients with polycythaemia vera were divided into the following groups: untreated polycythaemia vera; Received for publication 7 February 1969. treated polycythaemia (all these patients had been treated with radioactive phosphorus for varying periods of time⿳亠二口 up to 12 years); and spent polycythaemia (these patientš had all been treated with radioactive phosphorus and had developed a refractory anaemia). The cases of primary myelosclerosis had been diagnosed on peripheral blood findings; they had a chronic anaemia and there was nohistory of an antecedent polycythaemic phase. The diagnostic criteria of these patients (Table I) were out-lined in a previous publication (Roberts, Miles, and Woods, 1969).

Bone biopsies were obtained from the left anterior iliac 3 crest with a Sacker-Nordin trephine under local anaesthesia in a series of patients with polycythaemia vera and primary myelosclerosis. The cylinder of bone was fixed in barbitone-buffered $10 \%$ formol-saline and divided? transversely into two portions. The wholly cancellous $D$ portion was used for the preparation of undecalcified? sections; the other part was decalcified in neutral EDTA and sections were prepared from the endosteal end of theo specimen.

One decalcified section was stained with Ehrlich's haematoxylin and eosin for general histological assess- $\omega$ ment; another was stained by the silver impregnation technique of Gordon and Sweets and counterstained witho van Gieson's stain to assess the amount and distribution of reticulin and collagen.

Undecalcified sections were prepared and stained by the von Kóssa technique and counterstained with neutralo red. Osteoid measurements were recorded in three $\overrightarrow{\mathbb{D}}$ different ways using polarized light: (1) the number of $\tilde{\mathbb{P}}_{\text {}}$ laminae were counted; (2) the percentage length of trabeculae covered by osteoid was measured with an 
eyepiece graticule divided into $1 \mathrm{~mm}$ squares; (3) the area of osteoid as a percentage of total bone matrix (osteoid + calcified bone) was estimated using a Chalkley point-array graticule.

Full details of the technique of osteoid measurements have been recorded previously (Woods, Morgan, Paterson, and Gossman, 1968).

The percentage area of bone matrix in a section was estimated with a Chalkley array in a decalcified section using transmitted light.

The serum values for calcium, phosphorus, and alkaline phosphatase were measured according to Wootton (1964); if the alkaline phosphatase level was raised the heat stability index was estimated (Posen, Neal, and Clubb, 1965). Urinary hydroxyproline excreted over a 24-hour period was measured with a modification of the technique of Prockop and Udenfriend (1960).

\section{RESULTS}

MARROW MORPHOLOGY AND RETICULIN On examining the preparations stained for reticulin two main types of argyrophilic fibres were noted: one showed a branched pattern and was not refractile with polarized light; the second was smooth and wavy having the morphology of collagen and was refractile with polarized light. Neither fibre showed any staining property with van Gieson's stain. It was noted that the density of these fibres increased with bone marrow cellularity and that the second type of fibre although predominating in myelosclerosis was present in other myeloproliferative disorders and was not in itself a diagnostic feature (Roberts et al, 1969). However when the haematoxylin and eosin sections were examined, five cases $(26,27,28,29$, and 30) had an obviously different morphological appearance from the rest; they showed complete disorganization of normal marrow architecture due to marked proliferation of fibroblasts and reticulum cells and the presence of coarse bands of fibrous connective tissue. This appearance was considered to be the most important diagnostic criterion of myelosclerosis, and the cases with marrows showing this appearance are shown in Table $I$, and include the four cases of primary myelosclerosis and one case of 'spent' polycythaemia.

BONE STRUCTURE IN MYELOSCLEROSIS New bone formation was sought in all sections and was conspicuous in three cases $(27,28$, and 29$)$. In these the bone trabeculae were thickened, had irregular surfaces, and showed cement lines which were, in parts, poorly defined and slightly irregular in pattern, though this irregularity was not so marked as to suggest the arrangement in osteitis deformans. Osteoblasts were not numerous, but in the outer parts of the trabeculae plump osteocytes lay in
T A B L E I

OSTEOID AND BONE MATRIX MEASUREMENTS

\begin{tabular}{lllll}
$\begin{array}{l}\text { Case Initials } \\
\text { No. }\end{array}$ & $\begin{array}{l}\text { Area of } \\
\text { Osteoid (\%) }\end{array}$ & $\begin{array}{l}\text { Trabecular } \\
\text { Length } \\
\text { Covered (\%) }\end{array}$ & $\begin{array}{l}\text { No. of } \\
\text { Birefringent } \\
\text { Laminae }\end{array}$ & $\begin{array}{l}\text { Total } \\
\text { Matrix (\%) }\end{array}$ \\
\hline
\end{tabular}

\begin{tabular}{llll}
\hline \multicolumn{3}{c}{ Untreated polycythaemia vera } \\
1 & R.S. & 2 & 35 \\
2 & R.K. & -1 & - \\
3 & H.P. & -1 & - \\
4 & W.B. & -1 & - \\
5 & P.D. & -1 & - \\
6 & A.Sw. & 2 & 32 \\
7 & G.B. & $1 \cdot 5$ & 30 \\
8 & J.S. & 1 & 23 \\
9 & L.T. & 14 & 90 \\
10 & F.S. & $1 \cdot 5$ & 30 \\
11 & B.C. & 3 & 50
\end{tabular}

49
15
5
$\overline{21}^{13}$
10
10
$-^{1}$
6
10
16.4

Treated polycythaemia vera

\begin{tabular}{|c|c|c|c|c|c|}
\hline & & & & & \\
\hline 12 & A.Wa. & 4 & 46 & $1-2$ & 16 \\
\hline 13 & M.E. & 2 & 19 & $1-2$ & 16 \\
\hline 14 & N.S. & 2 & 13 & $1-2$ & 24 \\
\hline 15 & G.W. & 2 & 45 & $1-4$ & 26 \\
\hline 16 & J.B. & $0 \cdot 2$ & 3 & 1 & 23 \\
\hline 17 & H.B. & $2 \cdot 5$ & 15 & $1-2$ & 20 \\
\hline 18 & J.N. & 3.5 & 49 & $1-3$ & 20 \\
\hline 19 & A.C. & $3 \cdot 0$ & 37 & $1-3$ & 15 \\
\hline 20 & A.S. & 0.5 & 8 & 1 & 11 \\
\hline 21 & A.Wh. & 4 & 46 & $1-3$ & 18 \\
\hline \multicolumn{6}{|c|}{ Spent polycythaemia vera } \\
\hline 22 & E.B. & -1 & - & - & $17 \cdot 5$ \\
\hline 23 & C.B. & 0.2 & 2 & 1 & $19 \cdot 5$ \\
\hline 24 & W.R. & 1 & 10 & 1 & 13 \\
\hline 25 & L.O. & 1 & 36 & $1-3$ & 21 \\
\hline 26 & E.P. & $--^{2}$ & - & - & -1 \\
\hline \multicolumn{6}{|c|}{ Primary myelosclerosis } \\
\hline 27 & O.G. & -2 & - & - & 20 \\
\hline 28 & T.F. & $-^{2}$ & - & - & 49 \\
\hline 29 & M.L. & -2 & - & - & -1 \\
\hline 30 & O.P. & $6^{2}$ & 70 & $1-2$ & $15 \cdot 5$ \\
\hline \multicolumn{2}{|c|}{ Normal range } & $0-5$ & $0-45$ & $0-3$ & $>15$ \\
\hline
\end{tabular}

(Woods, Morgan, Paterson, and Gossman, 1968)

${ }^{1}$ These were not measurable either due to fragmentation of the section or to the inclusion of subcortical bone.

${ }^{2}$ Cases with histological changes of myelosclerosis in the marrow. Osteoid was not measurable in cases 26 to 29 for reasons explained in the text.

round or oval lacunae and were closer together than in the central parts of the trabeculae.

Examination of these sections in polarized light showed that most of the trabecular thickening was produced by the addition of woven bone to the surface, explaining the irregular surfaces of the trabeculae and the plump osteocytes. It was, however, difficult, when using polarized light, to distinguish between the advancing border of new bone formation and the fibrous tissue of marrow and impossible to measure osteoid. This was due to the fact that the refractile fibres of bone marrow were continuous with the fibres of bone matrix (Figs. 1 and 2), and this observation led to the conclusion that the new bone formation of myelosclerosis arises by ossification of preformed smooth, wavy, argyrophilic fibres in marrow. Ossification of branched non- 


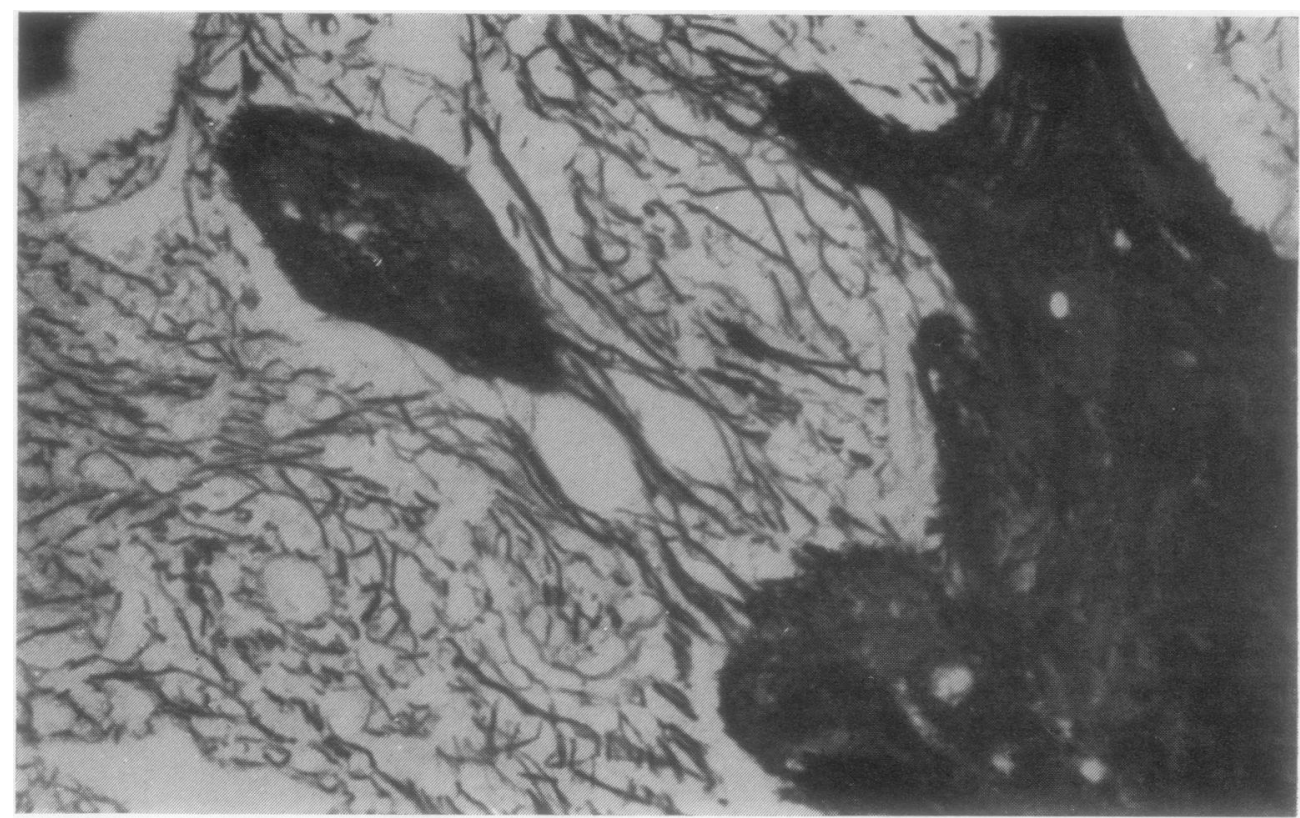

FIG. 1. Argyrophilic fibres in myelosclerosis, transmitted light $\times 300$.

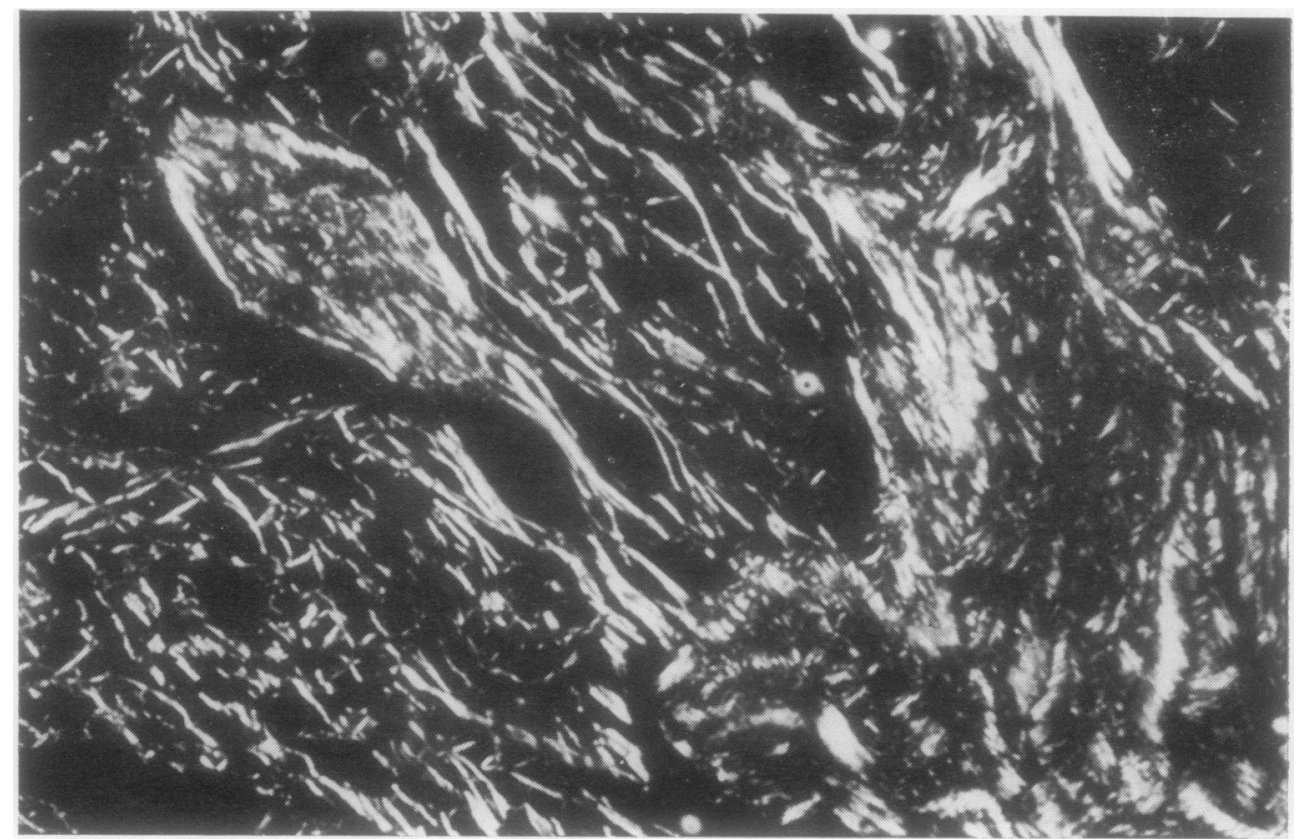

FIG. 2. The same field as is shown in Fig. 1 but using polarized light which demonstrates the continuity of the fibres of bone marrow and matrix $\times 300$. 
refractile reticulin fibres was not observed. In one case of myelosclerosis (case 30 ) the bone still had a predominantly lamellar construction and there was no demonstrable increase in the quantity of bone. In this case it was possible to measure the osteoid, which was slightly in excess of normal, due mainly to the increase of bone surface covered by osteoid but the amount of bone matrix was at the lower limit of normal.

BONE CHANGES IN POLYCYTHAEMIA VERA No new bone formation was observed in polycythaemia vera, and, as the figures in Table I show, there was no apparent increase in trabecular bone in the sections from polycythaemia vera: indeed some were osteoporotic as might be expected with the elderly age distribution of this group.

Osteoid measurements in the polycythaemic groups were within normal limits (Table I) except for a few cases, and then the increase was slight and mainly confined to the percentage of trabecular length covered. An exception was provided by case 9 in which measurements of the area and trabecular length of osteoid were notably increased. But it should be noted that, from measurements of total matrix, this patient was grossly osteoporotic and the amount of osteoid was measured as a percentage of total bone matrix. So in absolute terms the amount of osteoid must be much lower; and in this context the osteoid, when represented as a number of birefringent laminae, was within normal limits.

BIOCHEMICAL CHANGES Hydroxyproline excretion in all patients was normal, including those with gross myelosclerosis. Values for serum calcium and phosphorus were also entirely normal. Serum alkaline phosphatase levels were measured in 26 cases and these were raised in five cases though the highest value recorded was $30 \mathrm{King}$-Armstrong units. Heat stability indices on the cases with raised levels showed that the enzyme had its origin in bone (less than 0.2 ) in one case (treated polycythaemia vera, case 15); in liver (greater than 0.3 ) in two others (untreated polycythaemia vera, cases 6 and 9); and normal proportions of liver and bone enzyme (between 0.2 and 0.3 ) in the other two cases (one treated, case 20 , and one spent polycythaemia vera, case 23).

\section{DISCUSSION}

Myelosclerosis has been the subject of many publications but detailed descriptions of histology and histogenesis show wide variation.

There is little doubt that new bone is formed around preexisting trabeculae but is there an initial phase with osteoid formation, as Hartmann, Klima, Czitober, and Rieder (1959) suggest? Increased osteoid was found by Oeschlin (1956) in an intermediate phase in the development of myelosclerosis and by Leonard, Israëls, and Wilkinson (1957) in a late phase. However, Chapman (1933), Turnbull (cited by Vaughan and Harrison, 1939), Erf and Herbut (1944), and Rohr (1956) reported that osteoid was sparse. In this series osteoid has been measured in three different ways: the area of osteoid as a percentage of total bone matrix, the length of trabeculae covered, and the number of birefringent laminae. The present figures, with the exception of cases 9 and 30, are mostly within the normal range though several, particularly when measuring length of trabeculae covered, are at the upper limit of normal or slightly increased. An increased amount of osteoid may be due to osteomalacia or Paget's disease (Paterson, Woods, and Morgan, 1968) but there may also be a spurious rise in osteoid in osteoporotic bone, because of a reduction in bone matrix (Woods, Paterson, and Morgan, 1968). In any series of bone specimens from elderly patients, some can be expected to have abnormal osteoid estimations because of osteoporosis and possibly the early stages of osteitis deformans; case 9 had severe osteoporosis both histologically and radiologically.

Oeschlin (1956) described an initial stage of myelosclerosis in which there was marked osteoclastic activity and this was also reported in one of the cases of Beattie and Withey (1953). In the present series the only evidence is possibly that of past osteoclastic activity from the irregular contour of the cement lines; no multinucleate osteoclasts were identified on bone surfaces.

One explanation of the relationship between polycythaemia and myelosclerosis is that they arise from a common stimulus to the stem cell (Vaughan and Harrison, 1939; Dameshek, 1951; Wasserman, 1954). This is the concept of the 'myeloproliferative disorders' and accordingly there should be a proliferation of osteoblasts. This could not be corroborated by examination of the sections of the present series; indeed they were found to be sparse which is in agreement with the findings of Erf and Herbut (1944), Wyatt and Sommers (1950), and Oeschlin (1956). The serum alkaline phosphate level may be raised in some cases of myelosclerosis (Rohr, 1956) and it may be used as an index of osteoblastic activity, particularly if the heat stability index is below $\mathbf{0 . 2}$ (Posen et al, 1965). However, only one case (case 15) showed a raised level of alkaline phosphatase of bony origin but on examination of the bone biopsy there was no undue osteoblastic activity, marrow fibrosis, or new bone formation, though an occasional 
increase in the number of osteoid lamellae was seen. In two cases a raised alkaline phosphatase level was considered to be of hepatic origin; both these cases had appreciable hepatosplenomegaly, and this raised level was possibly associated with hepatic extramedullary haemopoiesis.

Both from the present series and reports in the literature (Carpenter and Flory, 1941; BlackSchaffer and Stoddard, 1953; Oeschlin, 1956; Rohr, 1956), there seems little doubt that although myelosclerotic fibrosis of the bone marrow can occur without new bone formation the reverse is not true. The wavy reticulin fibres termed 'pre-collagen' by Robb-Smith (1957) appear, from the polarized light examination, to undergo progressive ossification. This degree of organization of fibres seemed essential for the formation of new bone: ossification of branched, non-refractile reticulin was not observed. Under these conditions of ossification it was not possible to calculate the amount of osteoid. All the organized wavy fibres could, theoretically, ossify, and there was no demonstrable change in these fibres at the light microscope level of magnification to distinguish an advancing bone surface from a static one.

Our observations suggest that myelosclerosis begins as a fibroblastic proliferation which leads to the replacement of marrow by refractile wavy "precollagen' fibres. These fibres are progressively ossified by intramembranous ossification, which does not require that specialized osteoblastic cells should proliferate on the bone surfaces. In this way myelosclerosis differs from Paget's disease of bone and other diseases in which permanent sclerosis of cancellous bone is a feature.

We have recently noted a similar process in an osteosclerotic secondary deposit of carcinoma of breast in bone, and this type of ossification appears to be analogous to that seen in bony spurs forming. at tendon insertions ('rider's bones') and in some fibrosarcomata.

This is a further indication that the primary응 abnormality is in the fibroblast and that the new⿳亠口冋 bone formation in myelosclerosis is essentially a secondary and subsidiary phenomenon.

We wish to thank Dr J. R. Fountain and Dr C. J. Hayter $\vec{\circ}$ for allowing us to investigate patients under their care, and $\mathrm{Mr}$ Ian Salmon for the histological preparations. One of us (C.G.W.) is in receipt of a grant from the Medical Research Council.

\section{REFERENCES}

Beattie, J. W., and Withey, J. L. (1953). Brit. med. J., 2, 414.

Black-Schaffer, B., and Stoddard, L. D. (1953). Amer J. Path, 29,413

Carpenter, G., and Flory, C. M. (1941). Arch. intern. Med., 67, 489.

Chapman, E. M. (1933). Amer. J. med. Sci., 185, 171.

Dameshek, W. (1951). Blood, 6, 372.

Erf, L. A., and Herbut, P. A. (1944). Ann. intern. Mod., 21, 863.

Hartmann, G., Klima, R., Czitober, H., and Rieder, H. (1959). Wien Z. inn. Med., 40, 437.

Leonard, B. J., Israëls, M. C. G., and Wilkinson, J. F. (1957). Quart. J. Med., 26, 131 .

Oeschlin, R. J. (1956). Acta haemat (Basel), 16, 214.

Paterson, C. R., Woods, C. G., and Morgan, D. B. (1968). J. Pathe Bact., 95, 449.

Posen, S., Neale, F. C., and Clubb, J. S. (1965). Ann. intern. Med. 62,1234 .

Prockop, D. J., and Udenfriend, S. (1960). Analyt. Biochem., 1, 228.

Robb-Smith, A. H. T. (1957). In Symposium on Connective Tissues, edited by R. E. Tunbridge, p. 177. Blackwell, Oxford.

Roberts, B. E., Miles, D. W., and Woods, C. G. (1969). Brit. Jo Hacmat., 16, 75 .

Rohr, K. (1956). Acta haemat. (Basel), 15, 209.

Szur, L., and Lewis, S. M. (1966). Brit. J. Radiol., 39, 122

Vaughan, J. M. (1936). J. Path. Bact., 42, 541.

- , and Harrison, C. V. (1939). Ibid, 48, 339.

Wasserman, L. R. (1954). Bull. N. Y. Acad. Med., 30, 343.

Woods, C. G., Morgan, D. B., Paterson, C. R., and Gossman, H. H (1968). J. Path. Bact., 95, 441.

- Paterson, C. R., and Morgan, D. B. (1968) Orthopaedics, 1, 121

Wootton, I. D. P. (1964). Microanalysis in Medical Biochemistry, 4 th ed. Churchill, London.

Wyatt, J. P., and Sommers, S. C. (1950). Blood, 5, 329. 